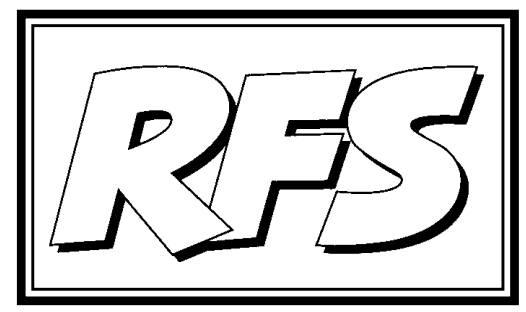

Revista de Fomento Social, 59 (2004), 823-840

\title{
El contexto local en la conciliación de vida familiar y vida profesional
}

Javier CALLEJO ${ }^{1}$

(PALABRAS CLAVE: CONCILIACIÓN, VIDA FAMILIAR, VIDA PROFESIONAL, POLÍTICA LOCAL, MERCADO LABORAL

KEY WORDS: RECONCILIATION, FAMILY LIFE, PROFESSIONAL LIFE, LOCAL POLICY, LABOUR MARKET)

\section{El aumento de la actividad femenina}

Hablar de actividad refiriéndose exclusivamente al mercado laboral tiene siempre el riesgo de olvidar toda aquella actividad que no se encuentra mercantilizada. Claro está, tal exclusión afecta fundamentalmente al trabajo reproductivo o doméstico y, en función del carácter patriarcal de la división

1 Profesor Titular de Sociología de la UNED. Agradezco encarecidamente los comentarios llevados a cabo por los evaluadores de la revista, que han ayudado a conseguir un mejor resultado final de este trabajo. 
del trabajo que toma en la mayor parte de las sociedades, afecta en especial a la actividad de las mujeres. Como se ha venido a reconocer (Esping-Andersen, 1999), más tarde que pronto, la actividad masculina y las instituciones que se crean alrededor del pleno empleo (masculino) reposan sobre esa actividad femenina no inserta en el mercado.

Precisamente por la relación de dominación que se encuentra tras la falta de tal reconocimiento de la actividad femenina, las mujeres se han ido inclinando por una mayor inserción en el mercado laboral. En España, también se ha registrado tal aumento de la actividad femenina. Ello a pesar de unas políticas que asumen la familia como principal regulador compensatorio de los desajustes en el mercado laboral y de políticas familiares supuestamente más destinadas a promover la natalidad, que la entrada de la mujer en el mercado laboral (Flaquer, 2000; Pérez Díaz y otros, 1998). Unas políticas que tienen consecuencias opuestas cuando las mujeres presentan una marcada decisión por entrar en el mercado laboral y los varones se resisten en la participación en la educación, cuidado de los hijos y las tareas domésticas, como es la disminución acelerada del número de nacimientos.

En 1976, la tasa de actividad femenina se encontraba alrededor del 28\%. Hace falta que pase un decenio, para que se supere el 30\% (1987), hasta situarse en el 44,66\% actual (III Trimestre de 2004, de la Encuesta de Población Activa). Aun se encuentra muy por debajo de las tasas de actividad entre mujeres adultas (más de dieciséis años) de los países del norte de Europa, que se encuentran alrededor del $70 \%$ desde hace varios decenios ${ }^{2}$. Conviene destacar aquí que la opción de la mujer española por la actividad laboral aparece como algo irreversible entre las generaciones jóvenes. De hecho, el salto puede calificarse de enorme. Mientras la tasa de actividad de las mayores de 55 años se queda en un $10 \%$, entre las que tienen entre 25 y 54 años llega a un $67,89 \%$ en el tercer trimestre de 2004.

Como muestra el Gráfico I, en la evolución reciente de la tasa de actividad femenina en España, pueden establecerse tres etapas. Una primera etapa hasta el referido año 1987, con un escaso aumento de la tasa de actividad femenina y, sin embargo, fuerte impulso del paro femenino, pudiéndose hablar de etapa de fuertes resistencias del mercado laboral a la inserción de la mujer, condenándola al desempleo. Una segunda etapa entre 1987 y

2 Islandia $79 \%$, Noruega $69 \%$, Finlandia $64 \%$, Suecia $76 \%$, Dinamarca $74 \%$. Datos referidos al año 2001. 
finales de 1994, con aumento constante de la tasa de actividad femenina y vaivenes en la evolución de la tasa de paro femenina, pues desciende al principio de esta etapa; para aumentar después. Una evolución cambiante que ha de relacionarse con los cambios en la regulación en la gestión de ayudas por desempleo, que se producen en el período. La tercera etapa, desde 1995 hasta la actualidad, donde se observa la evolución divergente de ambas tasas, con un coeficiente de correlación simple negativo muy alto $(-0,87)$. Una tercera etapa en la que parece que el mercado laboral acepta mejor a las mujeres y éstas, a su vez, se interesan más por el mismo al tener "más fácil" la entrada.

\section{GRÁFICO 1}

\section{Evolución de tasa de actividad femenina y tasa de paro femenino}

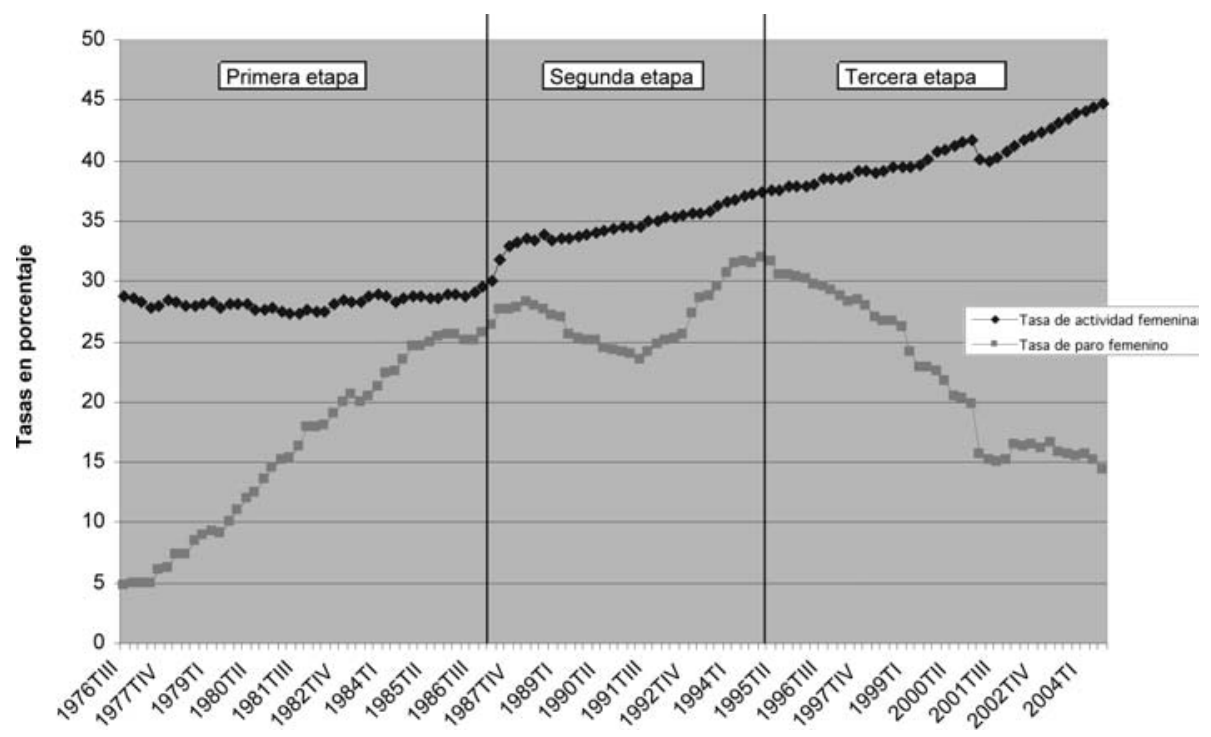

Trimestres EPA

Fuente: INE: Encuesta Población Activa.

Es decir, en la tercera etapa, que ya dura más de nueve años, parece ser el mercado el que llama a las mujeres. Aun cuando todavía no puede hablarse de ajuste entre la demanda del mercado y el deseo de las mujeres de inserción en 
el mercado laboral, como indican unas tasas de paro femenino de alrededor del 15\% (14,38\% en el tercer trimestre de 2004, según EPA), tampoco podría hablarse de rechazo del mercado laboral a la inserción de las mujeres, aun cuando, si se comparan con las tasas masculinas $(7,87 \%$ de tasa de paro masculino), cabe seguir hablando de discriminación, ya no sólo por el diferencial entre ambas tasas (actividad y desempleo) entre varones y mujeres, sino por las formas en que están ambos sexos en el mercado laboral.

La tercera etapa coincide con los años en que se empieza a hablar de la necesidad de conciliación de la vida familiar y profesional (CVFP en adelante). De hecho, en la actualidad de finales de 2004, la mayor parte de los ayuntamientos no han introducido explícitamente el concepto de conciliación como principio que guíe sus políticas, a pesar de que casi todos los que tienen más de cien mil habitantes ya se encuentran desarrollando su II Plan de Igualdad municipal y de que la Unión Europea adopta tal principio hacia la segunda mitad del decenio de los noventa ${ }^{3}$.

Ahora bien, la conciliación surge como problema al conectarse la inserción laboral de las mujeres con el descenso de la natalidad. De hecho, se observa cómo se pasa en tres lustros de una tasa de fecundad de 1,64 en 1985 a una de 1,15 en $1998^{4}$, repuntando a una de 1,25 en 2002, con la participación de la población inmigrante, situándose, en todo caso, entre las más bajas de Europa y del Mundo. Es decir, el descenso de la natalidad coincide con el período de aumento de actividad femenina, lo que, en principio, parece mostrar como poco coincidentes ambos ámbitos, el familiar y el laboral. Al menos, mientras el modelo de familia y/o el modelo de organización laboral no cambien.

Según la perspectiva que se tome, pueden encontrarse distintas-y no excluyentes-causas de tal aumento de la actividad femenina. Desde la perspectiva

3 El Tratado de Amsterdam (octubre 1997) y la Reunión Extraordinaria del Consejo Europeo sobre el Empleo de Luxemburgo (noviembre 1997) tienden a ser situados como los puntos de partida, pues en uno de los pilares del último -el destinado a reforzar las medidas sobre igualdad de oportunidades- se incluyen aspectos vinculados a la conciliación. La evaluación de la experiencia de lo que ha venido a llamarse "Proceso de Luxemburgo", que se realiza en 2002, pone énfasis en objetivos como la obtención de porcentajes mínimos de cobertura de plazas de guarderías. Ahora bien, es especialmente con la iniciativa comunitaria Equal cuando se pone la conciliación, junto con la segregación de los mercados laborales, como objetivo de las políticas públicas en España.

4 Fuente: Instituto Nacional de Estadística. 
de género, la inserción en el mercado laboral otorga una independencia a la mujer y una fuerza, a la hora de negociar los roles domésticos y familiares (Meil, 2004), prácticamente insustituibles, especialmente en países como España, donde no existen transferencias de recursos con carácter universal a los individuos en función de su situación familiar o sus asignaciones monetarias son muy escasas. Desde la perspectiva de los hogares, la aportación de los ingresos refuerza las disponibilidades para el consumo, de manera que se ha tenido en esta búsqueda de mejor posición en la sociedad de consumo una de las razones del cambio de norma familiar: desde el modelo -tachado de patriarcal- de un único ingreso varonil, al de dos ingresos ${ }^{5}$. En España, actualmente este modelo familiar con dos ocupados no constituye la mayoría, pero sí un ideal. Hay un $63,2 \%{ }^{6}$ de núcleos familiares en los que la mujer (en pareja o en solitario) no figura como activa, en el mercado laboral. Por ello, la conciliación tiene sentido en el contexto español como palanca para la extensión de la actividad, para facilitar la actividad femenina. No obstante, ya se percibe el cambio de tendencia en nuestro país ¿En qué familias, en función de la condición socioeconómica del hombre, es más fácil que la mujer no aparezca como activa? Están concentradas en los hogares de más edad: jubilados o parados (en el 84,6\% de estos hogares, la mujer no aparece como activa) y viudas (62,3\%). Los demás modelos de hogar tienen tasas por debajo de la media (por lo tanto, la tasa media se encuentra inflada por los hogares mayores). También es alto el referido porcentaje de inactivas en los tipos de núcleos familiares que cabe considerar en desaparición: empresarios agrarios sin asalariados $(59,9 \%)$, donde la mujer trabaja en el campo, pero no se declara; empresarios con asalariados (58,5\%); en la categoría resto de trabajadores de explotaciones agrarias (58,4\%); contramaestres y capataces de explotaciones no agrarias (58,3\%); operarios cualificados y especializados de explotaciones no agrarias (57,7\%). Ahora bien, en casi la mitad $(48,7 \%)$ de los hogares en los que hay madres con hijos, ellas tienen una ocupación laboral.

En esta relación de perspectivas, no pueden dejarse a un lado las transformaciones del propio sistema económico. Especialmente han de destacarse

5 Modelo denominado de varias formas, incluso como modelo postmoderno de familia (Meil, 1999)

6 Todos estos datos, como los que vienen a continuación sobre la ocupación en los hogares y núcleos familiares, proceden del Censo de Población y Viviendas 2001 (Instituto Nacional de Estadística). 
dos. Por un lado, el notable crecimiento del sector servicios. Si en el año que aquí se está tomando como punto de partida, 1976, el sector servicios representaba el $40,9 \%$ de la población ocupada y el $53,6 \%$ de las mujeres ocupadas, en el III Trimestre de 2004 representa el 64,7\% de la población ocupada y el $83,5 \%$ de la población femenina ocupada.

Por otro lado, la terciarización de la economía, especialmente como en el caso de la española, con una terciarización con amplio peso de los servicios de relación personal con el cliente (turismo, hostelería, etc.), se instituye como un modelo que requiere otro tipo de ajustes de tiempos, distinto al de una economía industrial. Valga el ejemplo de que mientras ésta exige turnos en continuidad, donde la maquinaria no deja de funcionar, y, así, obtener el máximo rendimiento de ésta y la máxima productividad de los trabajadores, sin mencionar la evitación de los costes de "nuevos arranques" que se producen en algunos sectores de esta producción industrial; en el modelo de atención personalizada al cliente se trata de un ajuste continuo a sus horarios fragmentados. El empresario ya no requiere un servicio continuo, sino flexibilidad para ajustar el empleo de recursos humanos a las variaciones de las demandas de los clientes. El almacenaje de los productos, ante su posible falta de salida, no es el recurso cuando hay que atender a las demandas personales del cliente. A éste no se le puede hacer esperar. La racionalización inserta en el modelo de acumulación flexible (Boyer, 1986) pasa por un ajuste de tiempos ad hoc a las temporalidades del mercado, por lo que la jornada laboral empieza a dejar ser la referencia, para pasar el número de horas al año a ser el caballo de batalla en la negociación colectiva.

Pues bien, esta terciarización exige flexibilidad de tiempos, las mujeres se introducen especialmente por los contratos a tiempo parcial. Parecen ajustarse mejor a la misma, aun cuando más desde las dificultades que encuentran para la obtención de otros tipos de contrato a tiempo completo, que desde la adaptación de la organización laboral a su organización personal y familiar?

7 Prácticamente la tercera parte $(31,5 \%)$ de las mujeres que trabajan a tiempo parcial lo hacen por no haber encontrado un empleo a tiempo completo; mientras que sólo el 7,8\% de estas mujeres que trabajan a tiempo parcial lo hacen por preferir esta modalidad, según los resultados de la Encuesta de Calidad de Vida en el Trabajo 2003 (Ministerio de Trabajo y Asuntos Sociales). Hay que resaltar que estos resultados varían significativamente de los arrojados por la EPA (tercer trimestre), donde el 18,34\% de las 1.168 .100 mujeres ocupadas a tiempo parcial lo hacen por no haber podido encontrar un trabajo a tiempo completo, mientras que el $9,90 \%$ lo hace por preferir esta modalidad ("no querer trabajo de jornada completa") y el $14,43 \%$ por obligaciones familiares. 
Hay que tener en cuenta que se percibe esa mejor inserción de la mujer en la terciarización flexible desde concepciones como: a) para la mujer, el empleo es algo complementario y secundario a sus funciones de crianza; b) la ocupación femenina como una manera de complementar los ingresos en el hogar; c) un paso de los segmentos con mayores dificultades de inserción laboral, como es el caso de la mujer en España, hacia otras formas de empleo más favorables. Es decir, el sistema productivo obtiene ventajas de la desigual distribución de papeles en los hogares: aumenta su flexibilidad extrayéndola de las familias y, en especial, de las mujeres.

Por último, la propia perspectiva del sistema social, que, por un lado, ha de dar salida a lo que es un notable aumento de la titulación educativa de las mujeres. Un sistema social que es incapaz de hacer uso de estos recursos humanos en su sistema productivo tiene un grave problema de eficiencia: invierte en recursos que posteriormente son rechazados. Pero es que, además y por otro lado, este sistema social ve envejecer su población y, por tanto, la necesidad de aumentar los contribuyentes para hacer viable los sistemas de pensiones. La inserción de la mujer en el mercado laboral se convierte entonces en una necesidad del sistema social.

\section{Sociedad en desigualdad}

Una sociedad que es incapaz de facilitar la CVFP genera desigualdad. No sólo entre sexos, pues lo que hace es reproducir o aumentar la existente, sino también entre hogares. Para justificar tal afirmación conviene señalar, entre otras dimensiones, que la inserción de la mujer en la actividad laboral dista de estar distribuida de manera homogénea. Las tasas de paro son mayores entre las mujeres que tienen hijos, lo que indica sus dificultades para la inserción laboral. Por otro lado, la tasa de actividad femenina disminuye entre las mujeres de hogares de clases populares, frente a la de hogares de clase media, cuando la categoría de clase social queda transformada en un indicador a partir de la categoría profesional del cabeza de familia. Para observar cómo actúan los procesos de desigualdad de los hogares y su relación con la CVFP, tal vez convenga detenerse en las trayectorias de inserción en el empleo. Pueden observarse dos clases de trayectorias tipo por su origen y a partir de la distinción que realiza Piore (1983) entre mercado primario y mercado secundario de trabajo. En la actualidad, la inserción directa en el mercado primario (estabilidad laboral, buenas condiciones de empleo, expectativas de carrera promocional con protocolos, etc.) aparece casi exclusivamente 
vinculada a los empleos en la Administración Pública. Algo que no es baladí para el asunto abordado: los resultados del Tercer Trimestre de 2004 de la Encuesta de Población Activa decían que el 26,74\% de las asalariadas lo hacían en el sector público, frente a un 16,75\% de los varones. Así, las mujeres constituyen ya más de la mitad $(51,55 \%)$ de los asalariados del sector público. La mayor preferencia de las mujeres por el sector público cabe explicarla por la articulación de procesos más transparentes -control público y cierta objetivización- en la selección de recursos humanos en el sector público y porque éste ofrece condiciones más estables, que parecen facilitar el salto hacia la propia estabilidad familiar y los márgenes para la CVFP, aplicándose los derechos de los trabajadores registrados en la legislación laboral para el logro de tal conciliación (permisos, flexibilidad de jornada, etc.), además de partir de unas condiciones relativamente mejores para la misma. De hecho, en la Administración ha habido una mayor disposición a intervenir en su organización para una gestión de los tiempos laborales más proclive a la conciliación, ya sea para facilitarla a sus empleados, ya sea para facilitársela al conjunto de los ciudadanos (Belloni y otras, 2000).

La otra forma de inserción es a través del mercado secundario, que, en la medida que se ha ido extendiendo, ha ampliado su variedad interna, recorriendo desde empleos en condiciones poco favorables para el trabajador (escasa estabilidad, pocos ingresos, nula carrera promocional) a empleos que significan el peldaño inicial hacia mejores condiciones, como ocurre con los empleos bajo categorías como las de contratos en formación o becas. En común, tienen algunos aspectos: temporalidad de los contratos o relativos bajos ingresos; pero las expectativas de los sujetos insertos en ellos son distintas. Sobre todo, son distintas las expectativas de salida de este mercado secundario, algo que especialmente se concreta en el caso de las mujeres. Cuando se trata del segmento referido en primer lugar del mercado secundario, una de las salidas es la dedicación al hogar y los hijos. La conciliación se resuelve dejando a un lado el mercado laboral, lo que, a su vez, supone la salida del peor segmento del mercado secundario. Así, más de cien mil mujeres dejaron su empleo por razones personales o responsabilidades familiares en 2002; mientras que sólo lo hicieron seis mil hombres ${ }^{8}$. Pero hay que resaltar que los abandonos femeninos se concentran en las categorías laborales menos favorecidas: el 30,8\% (tabla I) de los abandonos laborales femeninos se dan en

8 Datos ofrecidos por el Área de Estudios y Análisis del Consejo Económico y Social en 2003. 
la categoría de trabajadores no cualificados y el $26,8 \%$ en la de trabajadores de servicios de restauración, servicios personales, servicios de protección y vendedores de comercio, cuando suponen respectivamente el $18 \%$ y $23 \%$ del total de ocupadas. Por lo tanto, están más inclinadas a abandonar la ocupación laboral por razones personales o responsabilidades familiares las que se encuentran en las categorías inferiores de la estructura ocupacional. Sin embargo, sólo abandona el 2,6\% de las mujeres ocupadas como técnicas y profesionales científicas e intelectuales, mientras constituye esta categoría el $16 \%$ del empleo femenino.

TABLA 1

Ocupación de las mujeres que dejan el empleo por responsabilidades familiares o razones personales hace menos de tres años, 2002

\begin{tabular}{|l|r|}
\hline \multicolumn{1}{|c|}{ Porcentajes en vertical } \\
\hline Técnicos y profesionales científicos e intelectuales & 2,6 \\
\hline Técnicos y profesionales de apoyo & 9,4 \\
\hline Empleados de tipo administrativo & 11,2 \\
\hline $\begin{array}{l}\text { Trabajadores servicios restauración, personales, protección y vendedores } \\
\text { comercio }\end{array}$ & 26,8 \\
\hline Trabajadores no cualificados & 30,8 \\
\hline Resto de ocupaciones & 19,2 \\
\hline
\end{tabular}

Fuente: INE, EPA.

Las mujeres que utilizan el otro segmento para su inserción en el mercado laboral, al que podríamos denominar segmento superior, establecen otras estrategias. La salida del mercado secundario es el paso al mercado primario, casi siempre en clave de profesionalización. Si en el caso de las mujeres de clases populares puede hablarse de "paso hacia atrás", entendiéndose así la retirada del mercado laboral y la dedicación exclusiva a la familia; el paso hacia el mercado primario puede entenderse como "paso hacia delante" de este segmento de mujeres. Ahora bien, tal salto tiende a exigir diferir la maternidad, pues podría convertirse en un "problema" para la promoción hacia una profesionalización, a la que tiende ir vinculada una extensa dedicación temporal incompatible con el cuidado de los hijos. Hay que tener 
en cuenta que se suele tratar de hogares con dos carreras profesionales en consolidación y expansión en el período de la paternidad/maternidad. Si al retraso derivado de un largo período de formación, que ha de pasar -casi obligatoriamente- por los estudios superiores universitarios, se une el del paso por el mercado secundario, tenemos que el margen de edad para la maternidad se estrecha, pues es relativamente fácil alcanzar la treintena sin haber dado tal paso hacia el mercado primario. Ello sin contar con los diferenciales obstáculos posteriores que encuentra la mujer en su carrera profesional, consignados bajo el término techo de cristal, y que tienen su peso a la hora de llevarla a cabo.

Lo que aquí se plantea es que mientras los dos ingresos en el hogar, en condiciones comparativamente favorables, permiten acudir al mercado para paliar los problemas derivados de la ausencia de CVFP; en el otro supuesto, la retirada de la mujer del mercado laboral reduce los ingresos del hogar, ya no sólo durante los primeros años de maternidad sino, seguramente, para el resto de vida del hogar, dadas las dificultades para volver al empleo cuando pasan años desde que se dejó el último empleo. La conciliación en el primer tipo de hogares se obtiene parcialmente por la vía del mercado. En el segundo, se abandona la conciliación abandonando el mercado laboral. Es aquí donde cabe hablar de creciente desigualdad, salvo que medie la intervención del sistema público.

\section{La mediación pública local en las necesidades de conciliación}

Las Administraciones Públicas han puesto la CVFP entre uno de sus principales objetivos en materia de políticas sociales. Bien es cierto que el apoyo del sistema público a las familias varía notablemente de un país a otro, aun sin salir de la Unión Europea. Así, el gasto en protección social destinado a la familia (en términos del Sistema Europeo de Estadística de Protección Social, SEEPROS ${ }^{9}$ ) es del 2,7\% en España en 2000, mientras es del 8,2\% en el conjunto de la Unión Europea. El porcentaje de gasto español en familias, dentro del total de gasto por parte del Estado, se encuentra entre cinco y seis veces por debajo de países como: Luxemburgo, Dinamarca, Irlanda, Finlandia, Suecia o Alemania. De hecho, el dato español es el más bajo de la Unión Europea. Las vías de actuación son muy diversas y sólo a modo de síntesis se señalan las siguientes:

9 Para más información sobre el SEEPROS, véase Oriols (2002). 
- Políticas de sensibilización, basadas fundamentalmente en campañas de información destinadas a legitimar ciertos comportamientos y deslegitimar otros, en especial aquellos que se apoyan en la injusta atribución de las tareas y responsabilidades familiares y domésticas a las mujeres. Tales actuaciones pueden estar dirigidas tanto a:

- Familia/Sujetos, casi siempre articuladas en campañas publicitarias de carácter masivo o en actuaciones a través de centros territoriales: escuelas, centros municipales y, en general, en espacios de las administraciones públicas.

- Empresas, lo que va desde campañas más o menos masivas dirigidas a las organizaciones empresariales, hasta actuaciones que premian las mejores prácticas empresariales a favor de la conciliación.

- Através de la promoción de cambios en las regulaciones, principalmente en el ordenamiento laboral. No obstante, como antes, cabe diferenciar distintos ámbitos de aplicación de estas políticas:

— En el ámbito de la relación entre empresas y trabajadores, casi siempre en clave de regulaciones destinadas a facilitar la vida familiar de los trabajadores (permisos por necesidades familiares, facilidad para horarios más convenientes) y a impedir que la maternidad -o, en su caso, la paternidad- suponga un obstáculo en la trayectoria profesional de los trabajadores. No obstante, no han de dejarse a un lado los cambios legislativos que han llevado, en algunos países europeos, a la obligatoriedad de ofrecer servicios de guardería en empresas de cierto tamaño.

— En el ámbito de las relaciones familiares, ampliamente considerado como un ámbito privado, la regulación tiene mayores dificultades de llevarse a cabo; pero no deja de ser un posible ámbito de actuación.

- Promoción de acciones y servicios que faciliten la conciliación. A diferencia de la siguiente vía, se trata de acciones públicas destinadas a facilitar que el mercado o la denominada sociedad civil (voluntarios, ONGs) provea de servicios que apoyen la conciliación. Su ejemplo más relevante son los servicios personales de proximidad: guarderías, residencia y, en general servicios de cuidado de personas dependientes durante el tiempo en que los dos adultos de la pareja trabajan. Como dice Fraise: "la palabra mágica en la actualidad es la de 'proximidad'. Próximo significa íntimo y práctico, convivencial y útil” (Fraise, 2000:230). 
- Oferta de acciones-programas y servicios por parte de los distintos niveles del estado:

- Por ejemplo, acciones para facilitar el acceso/retirada de la mujer en el mercado laboral, casi siempre en clave de asignación de recursos monetarios: pensiones que permitan vivir fuera de la actividad laboral para las mujeres con hijos o recursos financieros para que las mujeres puedan contratar en el mercado servicios de proximidad, para dedicarse a la actividad laboral.

- Por ejemplo, extensión de servicios de proximidad y apoyo a la conciliación: guarderías y residencias públicas, servicios decuidado deniños tras el horario escolar (al modo de las doposcuola italiana), etc.

Cuando hablamos de servicios de proximidad para apoyar la CVFP, el ámbito local adquiere especial relevancia (Sánchez de Madariaga, 2002). Es el nivel del Estado de bienestar próximo por definición. Por lo tanto, también ha de ser el nivel más apropiado para el análisis de las necesidades de conciliación. Es lo que se desarrolla a continuación: las necesidades de conciliación de las cincuenta ciudades españolas con más de cien mil habitantes.

\section{Círculos de la conciliación}

Teniendo en cuenta que nuestra unidad de análisis es la ciudad y no los hogares o los individuos, se pone de manifiesto la existencia de dos círculos opuestos en relación a la actividad laboral de la mujer. Por un lado, un círculo que podría denominarse virtuoso que articula: una tasa de actividad femenina relativamente elevada en el contexto español, acercándose o superando el 50\%; unos ingresos per capita y familiares también relativamente altos; un desempleo bajo; $y$, por último, una estructura demográfica que no tiene una población dependiente excesiva presionando sobre las mujeres potencialmente activas para quedarse en casa a su cuidado ${ }^{10}$. Observando la Tabla 2, se encuentran en este círculo virtuoso ciudades como: Vitoria, Valencia, Castellón, Barcelona, Madrid, Palma de Mallorca, Sabadell, San Sebastián o Santander. En estas ciudades, la mediación a favor de la CVFP aparece estratégicamente dirigida a apoyar la creciente actividad laboral de

10 Para operacionalizar este concepto se ha utilizado el índice de presión sobre la mujer potencialmente activa: población dependiente (menores de quince años y mayores de sesenta y cinco años) entre número de mujeres en edad de trabajar (entre 15 y 65 años).

\section{RFS}


la mujer, facilitando la misma con servicios de proximidad, ya sea ofreciéndolos directamente desde el sistema público, ya sea desde la promoción para que sea el mercado y la oferta privada la que se encargue de atender tal demanda. Nos encontramos con un importante peso de hogares con los dos miembros adultos ocupados, lo que dificulta la atención y el cuidado de los hijos o de los ancianos, pero, por otro lado, con importantes ingresos, lo que se refleja en la renta per capita de la localidad. A pesar de que la mayoría de las ciudades referidas, con la excepción de Barcelona y Sabadell no presentan un índice de presión de la población dependiente sobre las mujeres potencialmente activas demasiado alto, tal presión existe, aun cuando podría ir articulada con otras demandas de servicios para el hogar no tan vinculados a tal población dependiente. Puede decirse que hay una potencial demanda solvente para estos servicios, que se incrustarían en el círculo virtuoso de la conciliación. Como puede observarse, es un círculo con una notable trascendencia económica, pues significa activar una base para mantener el flujo productivo de la localidad.

Por otro lado, existe un círculo que puede denominarse vicioso, pudiendo ser considerado el opuesto al anterior, pues marca registros de los indicadores referidos inversos. Es decir: tasa de actividad femenina relativamente baja; ingresos también bajos; un desempleo femenino elevado; y, por último, una estructura demográfica con un peso importante de la población dependiente. Se encuentran en tal círculo, localidades como: Sevilla, Málaga, Dos Hermanas, Murcia, Algeciras, Albacete, Cartagena, Córdoba, Jerez de la Frontera, Gijón, Huelva o Jaén. Aquí la mediación institucional a favor de la conciliación cobra otro sentido. Aparece, sobre todo, con dos objetivos: a) crear empleos entre mujeres, que presentan altas tasas de desempleo, de manera que la promoción de estos servicios adquiriría la forma de yacimiento de empleo; b) muy centrada en los servicios de proximidad de carácter personal: atención a población dependiente, ya que parece suponer un obstáculo importante para que una parte importante de estas mujeres den el salto hacia la actividad laboral.

Tras estos círculos, late la relación positiva entre tasa de actividad femenina y renta per capita (coeficiente de correlación simple del 0,41 ), lo que va en la dirección de aumentar las desigualdades referidas en el apartado anterior, aun cuando ahora entre localidades, puede proyectarse entre hogares. Asimismo, puede apreciarse la relación negativa entre tasa de desempleo femenino y tasa de actividad femenina $(-0,55)$, subrayando la evolución descrita en los últimos años para el conjunto nacional: a mayor actividad femenina, menos 
desempleo femenino, y viceversa. Por último, hay que destacar la relación negativa entre renta per capita y tasa de desempleo femenina, con un discreto coeficiente de correlación simple de $-0,46$. Pero más allá de estos débiles coeficientes de correlación, hay que señalar la relación entre presión sobre las mujeres potencialmente activas ejercida por la población dependiente y la tasa de actividad femenina.

El peso de la población dependiente desincentiva la actividad laboral femenina, salvo que se desarrollen servicios de proximidad específicos para atender tal población. Servicios que indirectamente apoyen tal actividad laboral femenina y, por lo tanto, la regulación. Para que la adaptación no venga de la reducción de hijos y del descenso de la natalidad, que aparece como la alternativa racional al aumento de la desigualdad entre los hogares, como forma de rebajar la presión sobre la inserción de la mujer en la actividad laboral y sus consecuencias sobre los ingresos, se requiere la mediación institucional en, al menos, el sentido de facilitar servicios asequibles para la CVFP, ya sea invirtiendo en servicios colectivos para ocuparse de la población dependiente, ya sea promoviendo servicios de apoyo particularizado a las familias o los proyectos de las empresas que, conscientes de la importancia que tiene el equilibrio entre vida familiar y vida profesional para sus empleados y, por lo tanto, para el conjunto de la organización, establecen ingeniosas medidas, como las referidas por Ariza Montes (2002).

¿Qué ocurre si no funciona el eslabón institucional? Especialmente el destinado a establecer la unión entre ingresos y presión demográfica sobre la mujer. Si los ingresos son bajos ¿hay que considerar que aumenta la presión demográfica sobre la mujer y empieza así, de nuevo, el círculo vicioso? Por el contrario, si los ingresos son altos ¿hay que considerar que la presión demográfica disminuye porque se pueden contratar servicios o que la mujer con ingresos retrasa o disminuye la maternidad y, así, realimenta, en lo inmediato, el círculo virtuoso? Desde tal punto de vista, la mediación institucional local, de proximidad, sería necesaria tanto desde un punto de vista como desde el otro. En el círculo vicioso, desde la perspectiva de las consecuencias a corto plazo, intentando cambiar el sentido del círculo. En el círculo virtuoso, desde la perspectiva de las consecuencias a largo plazo, pues una escasa presencia de hijos lleva al crecimiento relativo futuro de la población dependiente. La obtención del círculo virtuoso significa una mayor riqueza, incluso ciñéndonos sólo a las dimensiones económicas, de la localidad. 


\section{TABLA 2. Indicadores socioeconómicos de ciudades españolas de más de 100.000 habitantes}

\begin{tabular}{|c|c|c|c|c|}
\hline & Tasa desempleo femenino & Presión Mujeres & Renta p/c & Tasa Actividad femenina \\
\hline A Coruña & 15,54 & 79,70 & 8.772 & 40,63 \\
\hline Albacete & 17,17 & 88,15 & 8.519 & 35,48 \\
\hline Algeciras & 18,51 & 86,30 & 7.762 & 30,83 \\
\hline Alicante & 10,53 & 88,70 & 9.088 & 45,17 \\
\hline Almería & 16,45 & 88,01 & 10.175 & 38,80 \\
\hline Badajoz & 14,56 & 86,34 & 7.210 & 45,70 \\
\hline Badalona & 16,78 & 80,30 & 11.471 & 39,12 \\
\hline Barcelona & 9,90 & 96,90 & 11.471 & 42,11 \\
\hline Bilbao & 13,33 & 92,10 & 12.767 & 37,81 \\
\hline Burgos & 15,45 & 87,60 & 11.300 & 39,26 \\
\hline Cádiz & 14,26 & 78,80 & 8.512 & 35,36 \\
\hline Cartagena & 17,00 & 93,10 & 7.581 & 30,03 \\
\hline Castellón P. & 9,28 & 66,72 & 11.297 & 47,07 \\
\hline Córdoba & 19,52 & 88,40 & 9.520 & 35,32 \\
\hline Dos Hermanas & 26,32 & 78,40 & 8.512 & 37,10 \\
\hline Elche & 9,68 & 76,30 & 9.088 & 44,48 \\
\hline Gijón & 22,43 & 83,40 & 9.590 & 37,59 \\
\hline Granada & 16,26 & 87,78 & 9.250 & 36,26 \\
\hline Hospitalet & 14,30 & 82,50 & 11.471 & 40,00 \\
\hline $\begin{array}{l}\text { Huelva } \\
\end{array}$ & 21,16 & 87,30 & 9.250 & 34,77 \\
\hline Jaén & 23,48 & 91,90 & 9.250 & 35,09 \\
\hline Jerez F. & 19,53 & 83,80 & 7.762 & 31,52 \\
\hline Las Palmas & 16,00 & 78,80 & 9.702 & 45,27 \\
\hline León & 15,24 & 90,20 & 9.462 & 36,98 \\
\hline Lleida & 10,35 & 92,25 & 12.354 & 40,74 \\
\hline Logroño & 13,45 & 85,00 & 12.054 & 41,60 \\
\hline Madrid & 10,20 & 88,80 & 11.651 & 44,80 \\
\hline Málaga & 32,56 & 83,00 & 8.512 & 36,48 \\
\hline Marbella & 23,23 & 74,50 & 10.175 & 40,79 \\
\hline Mataró & 15,72 & 85,30 & 11.471 & 42,76 \\
\hline Murcia & 13,22 & 90,20 & 8.287 & 37,52 \\
\hline Ourense & 14,77 & 88,30 & 9.557 & 39,03 \\
\hline Oviedo & 15,77 & 82,20 & 9.590 & 40,21 \\
\hline P. Mallorca & 13,04 & 83,20 & 12.572 & 43,82 \\
\hline Pamplona & 12,79 & 87,60 & 12.741 & 41,84 \\
\hline S. C. Laguna & 14,90 & 75,95 & 9.371 & 46,30 \\
\hline S. C. Tenerife & 13,23 & 78,40 & 9.371 & 49,92 \\
\hline Sabadell & 12,87 & 91,20 & 11.471 & 41,84 \\
\hline Salamanca & 19,36 & 90,60 & 9.382 & 36,49 \\
\hline San Sebastián & 12,42 & 89,60 & 13.027 & 41,88 \\
\hline Santander & 11,47 & 96,52 & 10.637 & 42,55 \\
\hline Sevilla & 21,81 & 86,60 & 9.250 & 36,24 \\
\hline Sta. Coloma & 15,38 & 75,38 & 11.471 & 39,36 \\
\hline Tarragona & 12,40 & 85,00 & 11.397 & 40,12 \\
\hline Terrasa & 14,40 & 90,37 & 11.471 & 42,55 \\
\hline Valencia & 11,91 & 84,90 & 10.460 & 44,47 \\
\hline Valladolid & 18,67 & 80,80 & 10.691 & 37,37 \\
\hline Vigo & 17,27 & 77,60 & 8.670 & 39,62 \\
\hline Vitoria & 13,18 & 76,10 & 12.741 & 42,30 \\
\hline Zaragoza & 15,50 & 88,60 & 11.422 & 39,53 \\
\hline
\end{tabular}

Fuente: INE (renta per capita), fuentes estadísticas autonómicas y Anuario Social “La Caixa”. Año 2001. 


\section{Conclusiones}

Las mujeres quieren ser laboralmente activas, los hogares quieren que las mujeres se encuentren en el mercado laboral obteniendo un ingreso y el sistema económico quiere que haya hogares en que las mujeres estén ocupadas. Entonces, algo falla para que la incorporación de la mujer al mercado laboral no se produzca. Es el momento en el que se toma en cuenta el papel de la familia como base del sistema económico, después de algunos decenios de cierta ocultación. Se descubre, como dice Esping-Andersen (1999), el hogar, y, con él, la exigencia de conciliar vida familiar y vida profesional más allá de la división entre sexos, si los sistemas sociales avanzados quieren sobrevivir. Por lo tanto, la conciliación no es un problema de mujeres, es un agujero en la línea de flotación de la reproducción del sistema social, incluyendo el sistema económico.

Durante el cuarto de siglo (1948-1973) de progreso económico sobre un modelo de familia -modelo varón sustentador (Lewis, 1992)- fue la propia familia la que engrasó el motor del progreso (Daly, 1996:7). La familia puede seguir engrasando el progreso, si, a la vez, ella misma es engrasada.

Las políticas públicas en el nivel local que desarrollan la oferta de servicios de proximidad para la CVFP se han mostrado con un papel estratégico para el propio desarrollo de las ciudades y, por ende, para el conjunto de la sociedad. Pueden ser el engrase que necesitan las familias. Ahora bien, tal evolución de las políticas sociales requiere profundos cambios en la propia configuración del Estado de bienestar. El reto de éste es transformarse en un Estado de bienestar local y próximo. Un reto que cobra especial significado en nuestro país por, al menos, dos razones. Por un lado, la estructura fiscal se ha descentralizado, pero lo ha hecho desde la Administración Central a la Administración Autonómica, quedando todavía la descentralización hacia la Administración Local. Por otro lado, España destaca por el escaso peso que tienen los gastos destinados a las políticas familiares, dentro de los presupuestos estatales.

Desde el análisis aquí realizado, la intervención pública a favor de la conciliación tendría por objetivo fundamental transformar el denominado círculo vicioso (alto desempleo femenino, baja actividad femenina, peso de población dependiente y bajos niveles de renta) por el denominado círculo virtuoso (bajo desempleo femenino, alta actividad femenina, bajo peso de la población dependiente y relativamente altos niveles de renta), aun cuando esto no significa que la mediación a favor de la CVFP pudiera dejarse a un 
lado en este círculo virtuoso. Adquiere otro sentido y otras prioridades. Por otro lado, los logros con relación a tal objetivo, fruto de las políticas públicas y acciones destinadas a favorecer la CVFP, pueden ser evaluados a la luz de algunos de los indicadores utilizados. Los resultados de las medidas públicas a favor de la CVFP pueden evaluarse en función de las modificaciones producidas en los indicadores y, por lo tanto, en la transformación del círculo vicioso en círculo virtuoso.

\section{Referencias bibliográficas}

ArIZA Montes, J. A., (2002), "El dilema del equilibrio vida personal versus vida profesional: un enfoque de recursos humanos", Revista de Fomento Social, $\mathrm{n}^{\mathrm{o}} 225$, vol. 57 , enero-marzo.

Belloni, M-C, Boulin, J.Y, y JunTer-Loiseau, A., (2000), "Del tiempo de trabajo a los tiempos de la ciudad", en M. Maruani, Ch. Rogerat y T. Torns (dirs.), Las nuevas fronteras de la desigualdad. Barcelona: Icaria.

BOYER, R. (ED.), (1986), La flexibilidad del trabajo en Europa. Madrid: Ministerio de Trabajo y Seguridad Social.

Daly, K. J., (1996), Family Time. Keeping Pace in a Hurried Culture. Thousand Oaks (Cal.): Sage.

Esping-Andersen, G., (1999), Social Foundations of Postindustrial Economies. Nueva York: Oxford.

FlaQUeR, L., (2000), Las políticas familiares en una perspectiva comparada. Barcelona: Fundación La Caixa.

FraISE, G., (2000), "Servidumbre, empleos de servicio y democracia", en M.

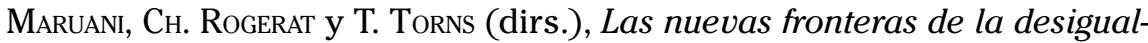
dad, Barcelona: Icaria.

Lewis, J., (1992), "Gender and the Development of Welfare Regime", Journal of European Social Policy, 2 (3).

MeIL, G., (2004), "Cambio familiar y maltrato conyugal a las mujeres" en Revista Internacional de Sociología, no 37, enero/abril 2004, pp. 7-27.

-(1999), La postmodernización de la familia española. Madrid: Acento Editorial.

ORIOLS, P., (2002), "El Seepros como instrumento de análisis social”, en Fuentes 
Estadísticas. Monográfico sobre Desigualdad y Pobreza, $\mathrm{n}^{\mathrm{0}}$ 63, marzo.

Pérez Díaz, V., Chulí, E., y Alvarez-Miranda, B., (1998), Familia y sistema de bienestar, Madrid: Argentaria.

Piore, M. J., (1993), "Notas para una teoría de la estratificación del mercado de trabajos", en L. ToHARIA (comp.), El mercado de trabajo. Teorías y aplicaciones, Madrid: Alianza.

SÁnCHEZ de MADARIAGA, I., (2002), "Las dimensiones urbanas de la conciliación de vida laboral y vida familiar", VV.AA., Conciliar la vida. Tiempo y servicios para la igualdad, Madrid: Consejo de la Mujer de la Comunidad de Madrid. 